Carmen Marimón Llorca

Universidad de Alicante

\title{
LOS DICCIONARIOS DE ECOLOGÍA Y MEDIO AMBIENTE EN ESPAÑOL O EL LÉXICO ENTRE LA CIENCIA Y LA CONCIENCIA
}

\section{Introducción: límites y limitaciones de los ámbitos de especialidad}

Es un hecho asumido que las obras Lexicográficas son un reflejo no sólo de las corrientes lingüísticas de las que son contemporáneas, sino de las actitudes y modos de pensamiento de la sociedad a la que se dirigen y de la que proceden. Los diccionarios de especialidad existen porque existe lo que se denomina «conocimiento especializado», un conocimiento «específico y marcado temáticamente [...] creado por especialistas para estructurar un determinado campo de conocimiento [...] propio de una comunidad de individuos que comparten y crean ese conocimiento» (Cabré y otros, 2001: 179). Los diccionarios de especialidad, pues, en la medida en que se proyectan sobre una parcela concreta del conocimiento y en tanto que están pensados como útiles para facilitar la comunicación entre los individuos especialistas en una determinada disciplina o ámbito epistemológico, están ligados a las necesidades de esa comunidad epistémica. Y de ninguna manera podemos pensar hoy en día que el conocimiento científico y su evolución sean algo aislado del resto de la sociedad (Galán Rodríguez, 2003:143).

Esta conexión entre conocimiento especializado y sociedad se hace particularmente evidente cuando las disciplinas con las que se trabaja, como es el caso de la Ecología o la Ciencias Medioambientales, son especialidades científicas de reciente creación, con límites aún difusos, pero de gran implantación académica y enorme popularidad social. El problema en este caso para el lexicógrafo o el terminólogo se complica extraordinariamente, pues a la dificultad que supone ponerle límites al conocimiento - ¿de qué se ocupa la ecología? ¿cuáles son sus disciplinas afines? ¿es lo mismo que el ecologismo?-, se suma, además, la de tener que decidir entre lo que pertenece -o ya no- al conocimiento especializado de esta materia, dado el constante proceso de retroalimientación que se da, en el caso de la Ecología, entre la ciencia y la sociedad. Ecología, ambiente, ecocrisis y otros vocablos son términos adoptados hoy en día -afirma Rodolfo Dirzo (1990: 48)- por una gama de individuos de Homo sapiens enfrascados en actividades tan diversas como el comercio, la docencia, el ecologismo o la ecología. La adopción de la «ecoterminología» -continúa explicando este autor- refleja, en muchos casos, la preocupación genuina por los llamados problemas ambientales, en otros, los ecotérminos son un obvio trampolín político. En este espacio entre la ciencia y la conciencia es en el que se encuentra esta disciplina, y lo que los diccionarios especializados reflejan es la inespecificidad de una materia absorbida y reclamada por tantas ramas del saber y del poder.

El objetivo de este trabajo es realizar un estudio valorativo general de los Diccionarios de Ecología y Medio Ambiente, tanto en lo que se refiere a la selección de las entradas, como al enunciado de las definiciones y a la información contenida en el artículo. Puesto que se trata de obras que han nacido al abrigo de esta nueva disciplina, su análisis nos permitirá observar algunas de las dificultades y limitaciones con las que se encuentra la elaboración de diccionarios y repertorios léxicos especializados, especialmente cuando, como ocurre en este caso, la materia es, al mismo tiempo, objeto de investigación científica y motivo de debate social. 
Metodológicamente, el trabajo se realiza comparativamente sobre las 8 obras lexicográficas especializadas en Ecología o alguna de sus disciplinas afines que se relacionan al final de este apartado. Son dos diccionarios en red, dos diccionarios terminológicos, tres diccionarios o gran diccionario y una base de datos terminológica. Para su estudio partimos de la idea señalada por numerosos estudiosos del tema (Ahumada 2001, Cabré 1993, Lorente 1999) de que las condiciones pragmático-textuales -destinatario, propósito o criterio de selección, entre otras- son esenciales para establecer los parámetros que condicionarán aspectos tan esenciales en los diccionarios de especialidad como la selección de entrada o la naturaleza de las propias definiciones. Por eso nos ha parecido necesario comenzar con una evaluación de los preliminares de cada una de estas obras con el fin de establecer dichas variables y así comprenderlas en la medida de sus propias intenciones. Con el fin de obtener una muestra representativa, hemos realizado una cala selectiva que ha consistido en analizar las veinte primeras entradas de la letra $\mathrm{M}$ de cada una de las 8 obras mencionadas.

Además de en la bibliografía correspondiente, nos basamos para este trabajo en las investigaciones previas del grupo EPA (Español Profesional y Académico) en el marco del IULMA (Instituto Interuniversitario de Lenguas Modernas Aplicadas de la Universidad de Alicante) del que formo parte.

Relación de obras analizadas:

- Colàs, Jaime (coord.) (1999): Gran Vox: diccionario de ecología y medio ambiente, Barcelona, Biblograf, 360 pág. (GVDEMA)

- Seoánez Calvo, Mariano (1996): El gran diccionario del medio ambiente y de la contaminación: definiciones en español con traducción de los términos al francés y al inglés, Madrid, Mundi-Prensa, páginas XXIII, 807 p. (GDMAC)

- Andrés Benito, Ana (1994): Diccionario del medio ambiente, Barcelona, EINIA, 313 págs (DMA)

- Martín Pérez, Antonio (2000): Diccionario terminológico de contaminación ambiental, Pamplona, EUNSA, 327 págs. (DTCA)

- Vicén Carreño, M. (1996): Diccionario de términos ecológicos, Madrid, Paraninfo, 173 págs. (DTE)

- Diccionario ecológico (www.DE) www.ambiente-ecologico.com/edicio-nes/diccionarioEcologico/diccionarioEcologico.php3

- Fausto O. Sarmiento (2001): Diccionario de ecología: paisajes, conservación y desarrollo sustentable para Latinoamérica. Ediciones Abya-Yala, Quito: CLACS-UGA, CEPEIGE, AMA [Primera edición digital de Diccionario de ecología, a cargo de José Luis Gómez-Martínez y autorizada para Proyecto Ensayo Hispánico, Octubre 2001]. (www. DE-S) www.ensayistas.org/critica/ecologia/diccionario/

- EURODIACUTOM. European terminology database (EURDAT) http://europa.eu.int/eurodicautom/Controller

\section{La ecología: los límites difusos de una disciplina nueva}

En su prólogo al libro Planeta azul, planeta verde, el científico Ramón Margalef afirma: «La palabra ecología que es, o era el nombre de una ciencia, y su adjetivo, ecológico, han entrado definitivamente en el lenguaje de la calle». En efecto, la ecología es una disciplina científica y académica, considerada una rama de la biología, de implanta- 
ción reciente en los estudios universitarios, ${ }^{1}$ y de muy rápido desarrollo y aceptación, cuyo objeto de estudio lo constituye todo aquello que tenga que ver con «los medios de vida naturales y las interacciones que se ejercen entre los seres vivientes y el medio en que viven, hábitat del que forman parte dichos seres». (www.DE). Es, según la definición holística «la ciencia del medio ambiente» (www. DE-S) por lo que no es de extrañar que estos términos, ecología y medio ambiente, aparezcan frecuentemente asociados.

Puede considerarse, por tanto, una transdisciplina, es decir, una ciencia en la que se abordan materias muy variadas pertenecientes a otras disciplinas cercanas, como la bioquímica o la geología, o mucho más lejanas conceptual y metodológicamente como el derecho. De hecho, la ecología tiene que ver, entre otros, con temas como la contaminación ambiental, el desarrollo sostenible, la biología, la legislación y el desarrollo jurídico, los movimientos sociales, las organizaciones no gubernamentales y algunas corrientes de pensamiento, además de con los conceptos de población, geología, clima, vegetación o territorio, entre otros. Es normal, pues, que en los prólogos de muchos de los diccionarios analizados se hable de la «multidisciplinariedad del tema del medio ambiente» (GVDEMA) o de la «pluridisciplinariedad del tema» (DMA).

Pero, por otra parte, como anunciábamos con la cita de Margalef, todo lo relacionado con la ecología y lo ecológico, con el medio ambiente y la contaminación, se ha popularizado con enorme rapidez y, gracias fundamentalmente a los medios de comunicación y a las organizaciones sociales, pero también a la percepción personal que los ciudadanos tienen del deterioro general de la condiciones ambientales de su entorno, la ecología y el medio ambiente se han convertido en una de las mayores preocupaciones de la vida actual. Así lo manifiesta el director general de la UNESCO, Federico Mayor Zaragoza, en el Prólogo a El gran diccionario del medio ambiente y de la contaminación: definiciones en español con traducción de los términos al francés y al inglés dirigido por Mariano Seoánez Calvo y bajo cuyo auspicio se realizó esa obra:

Si fuera posible aislar un solo factor de la compleja urdimbre de problemas que la $\mathrm{Hu}$ manidad afronta - es decir, si dichos problemas no estuvieran vinculados de manera inextricable, la defensa del medio natural sería, junto con la búsqueda de la paz, el que habría de recibir la absoluta prioridad. (GDMAC, p. VII)

El Diccionario de la RAE también se hace eco en su definición de esta naturaleza científico-social y activista de la ecología, como podemos observar en las tres entradas de la definición:

ecología: (De eco- y-logía).

1. f. Ciencia que estudia las relaciones de los seres vivos entre sí y con su entorno.

2. f. Parte de la sociología que estudia la relación entre los grupos humanos y su ambiente, tanto físico como social.

3. f. Defensa y protección de la naturaleza y del medio ambiente. La juventud está preocupada por la ecología.

1 El concepto de Ecología fue acuñado por el zoólogo alemán Ernst Haenkel hacia 1870 tras la aparición de la obra de Charles Darwin. Sin embargo, la eclosión de los estudios ecológicos se produce a partir de los años setenta del siglo XX cuando en 1971 se puso en marcha el MAB (Man and Biosphere), un programa de investigación de la UNESCO cuyo objetivo es «proveer los conocimientos científicos y el personal calificado necesario para el manejo racional y a largo plazo de los recursos naturales» (www.DE). 
Como consecuencia de esta universalización del problema y, con él, de la disciplina asociada, una parte importante de su vocabulario se ha instalado en la vida de los ciudadanos, que manejan y usan términos y conceptos como marea negra, recursos naturales, biodiversidad o reciclaje. Pero, además, $-\mathrm{y}$ sobre todo- la propia disciplina ha tenido que ajustar y recopilar su propio patrimonio terminológico, lo que ha significado no sólo definir los términos y conceptos que le son propios, como acción de respuesta, desarrollo sostenible, impacto ecológico, paisaje humanizado, sino adaptar a sus objetivos de estudio e investigación términos de otras materias como magnesio, ozono, magma, desalar, palustre, etc. De ahí que muchos diccionarios apelen a la constante renovación de la disciplina -y, por tanto, de su léxico- (GDVEMA) y a la «confusión terminológica» (DTCA) para justificar, como veremos, el sentido de su obra.

El hecho, pues, de que estemos ante una disciplina nueva y con un gran nivel de divulgación, tiene una consecuencia directa en los diccionarios o repertorios terminológicos de la materia, y es que tampoco en ellos están definidos claramente los límites del objeto de estudio, por lo que la selección de entradas está muy condicionada por la dirección que se dé a la disciplina -más jurídica, más estrictamente medioambiental, más ideológica, más orientada a la contaminación o a la biología-. Y esto está en el fondo de muchos de los problemas que nos hemos encontrado tanto en la selección de los elementos de la macroestructura como en el desarrollo de los artículos.

\section{La información contenida en los preliminares}

Un aspecto que confiere modernidad a la mayoría de las obras consultadas es el hecho de que, a excepción de la base terminológica EURODICAUTON, de uno de los diccionarios publicados en la red -Diccionario ecológico-, y del Diccionario de Términos Ecológicos, los cinco restantes contienen un prólogo en el que se precisan de manera concreta, como mínimo, los destinatarios, el propósito y el criterio de selección de las entradas. En algunos, además, se especifican las fuentes y se da otro tipo de información complementaria sobre la disciplina o sobre la propia obra como, por ejemplo, si aparecen los términos en otra u otras lenguas. ${ }^{2}$

Por lo que se refiere a los destinatarios, el GVDEMA es el que presenta un abanico más grande de posibles receptores pues está dirigido a un sector amplio de la población «(ESO, BACH, COU, $1^{\circ} \mathrm{s}$ cursos Univ.)», es decir a todos los «interesados sin formación científica». Es una obra de consulta, generalista, cuyo propósito explícito es «despertar mayor interés y concienciación por el medio ambiente» por lo que, como también indica en el prólogo, evitará «términos muy especializados (químicos)» que, sin embargo, serán habituales en otros diccionarios de la materia. Muy distinto es el público al que están destinados el GDMAC, el DMA y el DTCA. Se trata de obras dirigidas a personas relacionadas activamente con el objeto del diccionario (DMA), «Científicos, técnicos, miembros de organizaciones medioambientales. Investigadores, especialistas, estudiantes, profesores de la Universidad y legisladores» (DTCA) a los que se añaden «ingenieros, traductores, abogados, periodistas» (GDMAC) así como instituciones interesadas «Bibliotecas, empresas multinacionales multilingües» (GDMAC). Más allá de la consulta, estas obras que, además, en dos casos -GDMAC y DTCA-, incluyen equivalentes en otras lenguas, tienen

\footnotetext{
2 En la tabla 1 encuentra de forma esquemática la información contenida en los preliminares con especial atención a destinatarios, propósito, criterio de selección de las entradas, fuentes y otro tipo de información.
} 
propósitos más precisos que van desde los más estrictamente metalingüísticos como «ofrecer una nomenclatura apropiada [...] ayudar a la puesta al día [...] y retroajustar la terminología» (DTCA) a otros más prácticos como «facilitar los contactos internacionales en el ámbito medioambiental» (GDMAC). Un caso particular lo constituye el Diccionario de Fausto Sarmiento (DE-S), dirigido a «ecólogos latinoamericanos» y cuyo objetivo es «robustecer la disciplina en Latinoamérica cuyo material está en inglés» y «facilitar la reestructuración del estado del conocimiento ecológico en nuestros países». Se trata de un propósito casi educativo en el que el autor ha realizado un trabajo de adaptación, traducción y reformulación de conceptos procedentes tanto del inglés como de otros autores de habla hispana a lo que él denomina el «español latinoamericano». El resultado es un diccionario muy exhaustivo en el que hemos encontrado la mayoría de los términos que aparecen en los otros diccionarios.

El criterio de selección de las entradas también es muy dispar. Los dos únicos que dan información sobre el número de entradas son el GVDEMA y el GDMAC, pero ni estos ni ninguno de los que también tratan el tema-DMA, DTCA, www. DE-S- tienen ninguna pretensión de exhaustividad «voces útiles y prácticas», «enfoque generalista» «se prescinde»o «se incluyen productos químicos». Como veremos, sin embargo, no siempre resulta coherente la declaración de intenciones con la realidad de las definiciones.

Por otra parte, sólo tres de ellos, el GDMAC, el DMA y el DE-S dan información sobre las fuentes de las que proceden los términos. En general, estos son los muchos sectores implicados en los asuntos medioambientales, como legislación, corrientes filosóficas, acuerdos mundiales y europeos, expresiones cotidianas, managment (DMA). También se cita en el GDMAC la lista de los organismos consultados y es, además, el único que presenta un equipo explícito de redactores y una bibliografía -que también incluye aunque al final el DTCA-. Hay que señalar que el GDMAC se elaboró bajo los auspicios de la Fundación Desarrollo y Naturaleza (DEYNA) vinculada a la UNESCO y que es trilingüe por lo que sus criterios de redacción y ejecución son, con diferencia, los más precisos.

Es importante señalar también que algunas de estas obras contienen anexos o apéndices estrechamente relacionados con sus propios objetivos, así, el DMA que insiste mucho en su prólogo en las cuestiones legislativas, incluye en un anexo acuerdos de conferencias internacionales; por su parte, el DTCA, preocupado por la dispersión terminológica, añade anexos con acrónimos y abreviaturas en inglés y el DE-S tiene capítulos especiales «con términos no traducidos del inglés, raíces griegas y latinas» además de un anexo con Organizaciones de interés para la Ecología.

\section{Tipo de información}

\section{a) Las entradas}

Lo primero que nos ha llamado la atención en este aspecto es que sólo hemos encontrado una entrada que aparece en las ocho obras consultadas: marea negra, Macrófago, magma, maleza, manglar y marea aparecen en cuatro de ellas; madurez, en tres; y otras como macizo, magnesio, malpaís, malthus o MARPOL, en dos. Las demás, macla, magnetohidrodinámica, macolla, malatión, o mandato de Berlín, etc., sólo en uno de los diccionarios o repertorios analizados. Aunque la muestra que hemos utilizado no nos permita afirmaciones categóricas, pensamos que esta falta de correspondencia en las entradas es debida, precisamente, a la falta de concreción de la materia y a los objetivos difusos de las propias obras, lo que da lugar a una selección de términos muy poco coincidente $\mathrm{y}$, como vamos a ver, bastante poco rigurosa en algunos casos. 
Por lo que se refiere a las cuestiones formales, podemos señalar que la mayoría de las entradas seleccionadas son nombres comunes en singular, tal y como se recomienda en los manuales y trabajos de técnica lexicográfica y terminológica-magma, maleza, mantillo malformación-, pero también se encuentran otro tipo de entradas, como:

- nombres comunes en plural: maderas blandas, maderas duras, manglares (DMA, DTCA)

- nombres comunes especificados: marea negra, macizo antiguo, manto terrestre, maderas duras, maderas blandas

- nombres propios: Malthus

- siglas: $M A B, M A R P O L$

- Sintagmas terminológicos: Mandato de Berlín, Parámetro de Malthus, Teoría poblacional de Malthus, Población Malthusiama (en estas tres últimas la entrada es Malthus o maltusiana)

- Un adjetivo: macrófago

La presencia de los nombres comunes especificados está plenamente justificada pues al no aparecer en ningún caso subentradas, figuran como entradas conceptos que necesitan mayor precisión conceptual, hasta el punto de que algunos son prácticamente colocaciones - marea negra, manto terrestre-. Las siglas o los sintagmas son considerados por la terminología actual como USE (Unidades de Significación Especializada), por lo que su uso en este tipo de obras es muy frecuente e igualmente admitido. Otra cosa son las entradas en plural, cuya presencia no se justifican más que por el descuido de los autores, pues no se trata en ningún caso de lexicalizaciones; es el caso, por ejemplo, de manglar, que aparece en singular en cuatro diccionarios y en uno en plural, manglares.

Como señalamos más arriba, en algunos de los Diccionarios analizados se precisa el número y se acota el ámbito de procedencia de las entradas (ver tabla 1). Así, en el GVDEMA se dice que «se prescinde de términos muy especializados (químicos)» y que el Diccionario «está centrado en las palabras propias de la ecología y el medio ambiente». Por su parte, el GDMAC, presenta una «selección de voces más útiles y practicas»e «incluye productos químicos». Cuando contrastamos las definiciones de magnesio que realizan ambos diccionarios, resulta que la del GVDEMA que, teóricamente, no incluye términos químicos, proporciona tanta información especializada o más que el GDMAC, dirigido a científicos, docentes, etc.

magnesio $m$. QIM. Metal cuyo símbolo es Mg, su número atómico 12 y su masa atómica relativa 24,312, maleable, de color y brillo plateado, poco resistente, y que arde generando una luz brillante y clara. Constituye un macronutriente* de gran importancia para los vegetales, y se utiliza en la fabricación de fármacos, señales luminosas y en la fotografía. (GVDEMA)

Magnesio. Mg. Elemento de número atómico 12. Moderadamente duro. Es el metal más ligero. Soluble en ácidos. Inflamable.

F: Magnésium

I: Magnesium (GDMAC)

Con respecto a la selección de entradas en relación con los límites de la disciplina, el DMA y el DTCA indican en sus preliminares que van dirigidos a destinatarios más o menos especializados y, en particular, el DTCA destaca que está orientado a «concretar 
la nomenclatura a utilizar en contaminación ambiental». Así, en el DMA se dice que se han incluido «los términos más importantes», y en el DTCA parten de «la noción de contaminación ambiental, tomada en un sentido amplio», pero con el fin de «ofrecer una nomenclatura apropiada con la definición de los términos más usuales en contaminación ambiental». Sin embargo, como hemos podido comprobar, en la definición de marea negra de este último no hay ninguna mención del peligro -contaminante- que esta supone, mientras que todos los demás, incluido el DRAE y a excepción del www.DE-S, sí lo hacen:

\section{1) DTCA}

Marea negra (blach tide). Petróleo descargado de modo natural o por accidente y que flota sobre el agua como una masa discreta que es transportado por los vientos, corrientes y mareas.

\section{2) DMA}

Marea negra: Masa de petróleo vertida al mar que flota y es transportada por vientos, corrientes y que puede causar graves daños, sobre todo al llegar a la costa.

\section{3) DTE}

\section{Marea negra}

Capa de petróleo o combustible, de grosor variable, que flota sobre la superficie del mar como consecuencia de un vertido al mismo. Es absolutamente perjudicial para la flora y la fauna. La investigación está tratando de diseñar ciertas bacterias capaces de eliminarla. Vid. Accidente petrolífero.

4) W.DE-S

MAREA NEGRA. BLACK TIDE. Llegada a las playas de inmensas mesas de petróleo que flotan sobre el océano como producto de accidentes en el transporte de los buque-tanques petroleros, rotura de oleoductos submarinos y explosión de la flora y fauna marina costanera.

5) Www.DE

MAREA NEGRA. Capa de petróleo, generalmente escapada de manera accidental de un petrolero o de un pozo submarino, que flota en la superficie del mar o de un curso de agua y alcanza la orilla, ocasionando graves perjuicios en la fauna y el paisaje.

\section{6) RAE}

$\sim$ negra.

1. f. Masa de petróleo vertida al mar, que puede causar graves daños, sobre todo al llegar a la costa.

\section{b) Tipo de información contenida en el artículo ${ }^{3}$}

Ninguna de las obras analizadas incluye la etimología, algún tipo de información gramatical y normativa o ejemplos de uso. Resulta sorprendente que esta ausencia se dé incluso en Diccionarios que tienen como objetivo, como hemos visto, la fijación terminológica o la adaptación del léxico. En cuanto al nombre científico, aparece - de forma

3 En la tabla 2 se encuentran marcados con valores S/N (Sí/No) la siguiente selección de criterios: etimología, nombre científico, categoría gramatical, marcas temáticas de subespecialidad, información gramatical, equivalente en otra lengua, sinónimos en la misma lengua, información normativa, definición, ejemplos, iconoestructura. 
arbitraria en el interior del artículo- sólo en uno de los Diccionarios, el www.DE-S, al referirse, por ejemplo, a una clase o tipo de manglar: «En el Ecuador son comunes el mangle rojo (Rhizophora mangle) y el negro (Avicennia nitida)». Tampoco es frecuente el uso de sinónimos que sólo encontramos en el www.DE-S en dos casos, en uno de ellos explícitamente:

Malthusiana-Población. MALTHUSIAN POPULATION. Población que ha alcanzado tal estado de equilibrio en el número de individuos que, la distribución de la población total en clases de edad, se mantiene indefinidamente igual a sí misma; dicha población recupera sus características aunque experimente perturbaciones pasajeras. (Sinónimo: población estable).

Y en el otro, en el interior del artículo, entre paréntesis, sin ninguna especificación:

maleza. WEED. Clasificación de formas de vida vegetal para plantas herbáceas (malas hierbas) que se encuentra asociada a los terrenos baldíos y sembríos descuidados, constituyendo un serio problema para el agricultor, quien utiliza medios de eliminación de malezas.

Por su parte, el GVDEMA, es el único diccionario que incluye entre la información del artículo la categoría gramatical y las marcas temáticas de subespecialidad. Así se puede comprobar en el siguiente ejemplo:

maleza f AGR. y BIOGEOG. Abundancia de malas hierbas en un campo de cultivo, que infligen un perjuicio a los sembrados, o bien en un campo inculto. II BIOGEOG. Vegetación densa arbustiva, como los jarales y zarzales.

Llama la atención que esto ocurra, precisamente, en una obra divulgativa y que, sin embargo, esté ausente esta importante información en trabajos teóricamente más especializados y para especialistas que, por otra parte, sí dan cuenta de la nómina de disciplinas con las que se relaciona la materia de la obra y que, además, tienen entre sus propósitos facilitar los contactos internacionales o retroajustar la terminología. Como señalaremos en las conclusiones, dudamos mucho de que un diccionario sin información lingüística pueda cumplir algún tipo de objetivo comunicativo.

Otra importante cuestión tiene que ver con la inclusión de equivalentes en otras lenguas. Además del Eurodicauton, cuya finalidad es facilitar la traducción de términos a varias lenguas de la Unión Europea, ofrecen equivalentes el GDMAC -en francés y en inglés- el DTCA -en inglés- y el DE-S también en inglés. Mostramos un ejemplo de cada caso:

Madurez. Estado en el que un ser ha llegado a su desarrollo completo y se halla en la plenitud fisiológica.

F: Maturité

I: Maturity

Marea negra (black tide). Petróleo descargado de modo natural o por accidente y que flota sobre el agua como una masa discreta que es transportada por los vientos, corrientes y mareas.

madurez. MATURITY. Estado de organización avanzada de los componentes del sistema. 
Sólo el GVDEMA presenta iconoestructura lo que supone la inclusión esporádica de alguna ilustración -básicamente fotografías-.

\section{c) La definición: observaciones de tipo formal}

En la mayoría de los casos, la definición respeta el número gramatical del definido, pero se encuentran también casos como los siguientes:

- definición en singular cuando la entrada es en plural:

Manglares: Terreno que....

- definición en plural cuando la entrada es en singular:

Materia orgánica: Residuos de alimentos...

Macroalimento. Macronutrient. Elementos y compuestos...

Macrofago. Macrophage. Células fagocíticas...

Macrófago: Animales que se nutren...

En la mayoría de los casos, la categoría gramatical con la que se inicia la definición es la misma que la del término definido, sin embargo encontramos:

- entrada nominal y definición encabezada por verbo:

Maderas duras: Son árboles

- entrada nominal y definición mediante participio:

Marea Negra. Black Tide. Llegada a las playas...

- entrada nominal y definición encabezada por un artículo definido o indefinido:

Macronutrientes (macronutrients). Los elementos generalmente

reconocidos...

Mandato de Berlín (Berlin Mandate). Una resolución negociada en la...

MAB. MAB. El programa biológico internacional (IBP) estableció...

MALTHUS, TEORÍA POBLACIONAL DE. MALTHUS'

POPULATION THEORY. La población aumenta....

Se trata de pruebas de gran impericia y de verdadero descuido de la técnica lexicográfica. El DMA, DTCA, DE-S son los que redactan la definición de forma más arbitraria y descuidada mientras que el GDVEMA, GDMAC demuestran, como indican en el prólogo, tener equipos de redacción con criterios más unificados.

\section{d) La definición: tipos y características}

La totalidad de las definiciones analizadas en estos diccionarios son -o intentan serdefiniciones analíticas de tipo perifrástico «sustancial», «inclusivo»o «aristotélico» pues tratan de responder a la pregunta ¿qué es el definiendum? mediante una perífrasis, es decir, «una expresión compleja que refleja los rasgos que componen el sentido de la unidad definida" (Martínez Linares 2006). Son estas las definiciones que la lexicografía denomina propias pues se realizan en la llamada metalengua de contenido. Muchas definiciones de este tipo se realizan mediante procedimientos de hiperonimia. Así, un macizo antiguo es un conjunto montañoso, el magnesio es un metal, un macrófago es una animal, las maderas blandas son árboles, el manganeso es un mineral metálico, la magnetohidrodinámica es una técnica.

Otras veces, el hiperónimo se refiere a una clase o tipo a la que pertenece el definido; así el manglar es un [tipo] de formación vegetal, o un [tipo] de ecosistema de características complejas, malpaís es una [clase o tipo] de terreno y las maderas duras son árboles 
de [tipo] planifolio. En algunos casos, el hiperónimo no es un nombre concreto sino un abstracto mediante el cual se designa la cualidad o el concepto bajo el cual es posible incluir el definido. Es el caso de manglar que es definido como una asociación vegetal, el magnetismo, que es una propiedad que tiene la piedra imán, o la maleza que se define como abundancia de hierbas.

También se utilizan procedimientos metonímicos según los cuales el definido es una parte de un elemento nombrado en la definición. Así, la madera es la parte sólida leñosa de los árboles, el mantillo es la capa superficial del suelo, o el estrato superior del suelo, el manto terrestre es la capa de la tierra entre el núcleo y la corteza. En estos casos, la cuantificación depende de la naturaleza de la materia pues en unos casos el objeto podrá dividirse en partes, pero en otros será en capas (el manto terrestre), en estratos (el suelo), en fases o estados (el desarrollo).

Con cierta frecuencia se utiliza la definición por extensión, pero siempre en combinación con los procedimientos analíticos. Así, la maleza es una vegetación densa (análisis) como los jarales y zarzales (extensión) y un macronutriente es un compuesto nutritivo (análisis) como son, p. ej., el potasio, el nitrógeno, el oxígeno, etc. (extensión).

Lo mismo ocurre con la definición mediante síntesis en algunos diccionarios que, junto con el procedimiento analítico, incluyen información enciclopédica de tipo teleológico - la madera es la parte sólida leñosa de los árboles (análisis) que se utiliza en la fabricación de mobiliario (finalidad) -o genético- un macizo antiguo es un conjunto montañoso (análisis), de la era Paleozoica (origen), que durante la orogenia alpina, lejos de plegarse, se levantó y/o deformó en abombamientos de enorme radio de curvatura, o se fracturó y originó horsts y fosas tectónicas (causa).

Hay alguna definición que consiste en una traducción parafrástica del término, especialmente cuando éste es un compuesto culto procedente del griego. Son definiciones cercanas a las morfosemánticas, en las que se utiliza el aporte semántico de las partes del propio definido para elaborar la definición. Así macrófago se define como referente al animal que puede nutrirse de organismos de tamaño tanto o más grande que el suyo; animales que se nutren de presas muy grandes con relación a su tamaño. Se da el caso curioso de que en un diccionario se entiende equivocadamente el significado de uno de los elementos del compuesto -macro- y así se define como animal que se alimenta de presas de tamaño igual o algo menor al suyo.

Se dan muy pocas ocurrencias de utilización del definido en la definición, es el caso sin embargo de macrófago al que se describe como células fagocíticas.

\section{Conclusiones}

Como hemos podido observar, los diccionarios de Ecología y Medio Ambiente en español en la actualidad necesitan una seria y rigurosa puesta al día en todas y cada una de sus fases de elaboración si quieren ser realmente considerados como diccionarios de especialidad. En general se trata de obras más bien divulgativas, que quieren hacer compatible, como anunciábamos en el título, la ciencia con la conciencia; en otras palabras, cubrir las necesidades y las inquietudes de la sociedad con respecto al tema de la ecología pero, al mismo tiempo, dar respuesta a las exigencias de precisión terminológica de los especialistas. Así, una misma obra se presenta como dirigida tanto a un miembro de una ONG, como a un profesor universitario o a un científico; y con este punto de partida ni el enfoque, que condiciona la selección léxica -esencial en las obras especializadas-, 
ni la información contenida en los artículos puede tener una orientación coherente. Estamos ante trabajos que desde la presentación dan muestra de una gran heterogeneidad en todos los sentidos pues los términos ecología, medioambiente y contaminación, acompañados de las denominaciones diccionario o diccionario terminológico se utilizan en los títulos para referirse a campos de estudios y a métodos de trabajo diferenciados.

En cuanto a las entradas, la disparidad que hemos observado en la selección -sólo una coincidencia entre las veinte primeras entradas de la letra M, marea negra- es prueba del otro gran problema con el que se enfrenta la elaboración de este tipo de diccionarios y es el de los límites de la propia disciplina. No hay acuerdo sobre qué elementos químicos ni qué conceptos físicos, biológicos, geológicos, legales o sociopolíticos forman parte de ésta y deben, por tanto, incluirse. Esto ha dado lugar a que se haya realizado una selección en muchos casos arbitraria y poco o nada consistente. Por ejemplo, magnetohidrodinámica sólo se incluye en el GDMAC, donde también está la entrada magnetismo, y en el DTCA, donde no se encuentran magnetismo ni magnético. En los demás diccionarios se dan distintas combinaciones, como que aparezcan otros derivados -magnetosfera- o ninguna entrada referida al concepto. Se da el caso, además, de que este término sí está en el DRAE, donde tiene la marca Fis. Es evidente que la cercanía con otras áreas de conocimiento, como la contaminación o la biodiversidad e incluso la indeterminación en las diferencias entre los términos medio ambiente y ecología con los que se da título a muchas de estas obras, hacen muy difícil tanto una selección rigurosa de entradas, como la determinación de las USE lingüísticas y no lingüísticas que corresponden, dejando a los responsables de elaborar los diccionarios, la tarea de definir los límites de la disciplina. Si bien esto último supera con mucho las funciones de quien tiene por tarea elaborar una obra lexicográfica de especialidad, sí sería exigible a los equipos lexicográficos un mayor rigor formal tanto en los criterios de organización de la macroestructura como en la elaboración de los artículos, pues el descuido del que hemos dado pruebas en este trabajo va en evidente detrimento del valor que deben tener estos trabajos como instrumentos para la comprensión y la producción lingüística en una determinada área de conocimiento.

Por lo que se refiere al tipo de información contenida en el artículo, la falta de definición clara de propósito y destinatarios y la indeterminación de la disciplina vuelven a estar en el eje de los muchos problemas que hemos encontrado en este aspecto. Ninguna de las obras analizadas incluye la etimología, algún tipo información gramatical y normativa o ejemplos de uso. Parece olvidarse ahora que un diccionario, en cualquier caso, está formado por «unidades de naturaleza lingüística» y que como tales deben de ser tratadas (Santamaría, 2008: 8). Esta ausencia de información lingüística resulta aún más llamativa en los diccionarios que se autodenominan como terminológicos, pues la terminología lleva ya algunos años reclamando, precisamente, una orientación comunicativa (Cabré, 1999, 2001, 2002) y un tratamiento lingüístico de sus unidades orientado a la producción. Tal y como están planteados, este tipo de diccionarios ofrecen muy poca y pobre información con la que facilitar el uso de los términos seleccionados y definidos en el proceso de producción discursiva que necesita llevar a cabo un hablante especializado. De esta manera, la dirección onomasiológica que debería orientar estos diccionarios especializados de corte terminológico está claramente restringida por las limitaciones de la información que proporcionan.

Los aspectos formales de la definición son, una vez más, prueba de gran impericia y de verdadero descuido de la técnica lexicográfica. El DMA, DTCA, www.DE-S son los que redactan la definición de forma más arbitraria y descuidada, mientras que el 
GVDEMA y GDMAC demuestran, como indican en el prólogo, tener equipos de redacción con criterios más unificados. En cuanto al modelo de definición, la mayoría, como hemos visto, son de tipo analítico hiperonímico, aunque abundan también las definiciones por metonimia. También por lo que se refiere a la definición hay que señalar un exceso de información enciclopédica, muy difícil de evitar, por otra parte, en los trabajos lexicográficos de especialidad, como se ha señalado en muchas ocasiones (García Palacios, 2002: 41). Sin embargo, tal y como el propio García Palacios (2002: 42) señala, el problema no es sólo la presencia de este tipo de información sino el hecho de que ésta se reparta de forma irregular en los artículos de un mismo diccionario en función del interés de la entrada, dando lugar a microestructuras muy poco unificadas. Es el caso del DTE del que mostramos las definiciones de manto terrestre, de tipo metonímico y manglar, con abundante información enciclopédica:

Manto terrestre: Capa de la tierra entre el núcleo y la corteza; su límite superior es la discontinuidad de Mohorovicic y el inferior la discontinuidad de Gutemberg.

Manglar: Formación vegetal de especies halofitas propia de regiones litorales de la zona tropical. Es uno de los hábitats fundamentales para la reproducción de los peces, a la par que reduce los efectos de la erosión. Su progresiva disminución por tala para la obtención de la leña y la pulpa, en beneficio de criaderos de peces y mariscos o para edificación y cultivo, provoca una destrucción de la zona costera y reducción de la pesquería.

En otras ocasiones, la información que proporciona la entrada coincide casi completamente con la del DRAE, aún tratándose de un concepto incluido en un diccionario especializado, como es el caso de maltusianismo, cuya ortografía, por cierto, varía entre maltusianismo o malthusianismo en las distintas obras:

Maltusianismo. Teoría propuesta por Malthus que suponía que el crecimiento de una población seguiría una progresión geométrica y el de los alimentos una progresión aritmética, dando lugar a escasez y a crisis periódicas (DMAC)

Maltusianismo: Conjunto de teorías económicas de Thomas Malthus, economista británico de fines del siglo XVIII basadas en que, según él, la población tiende a crecer en progresión geométrica mientras que los alimentos sólo aumentan en progresión aritmética (DRAE).

En definitiva, los diccionarios de ecología y medio ambiente son, hoy en día, un reto para la lexicografía de especialidad en español. El primero de ellos es definir claramente los destinatarios y establecer unos fines concretos que den una dirección coherente a la obra; eso permitirá elaborar buenos diccionarios de divulgación y buenos diccionarios especializados que den respuestas a las necesidades de la comunidad científica pero también a la sociedad, que reclama cada día más información sobre el mundo de la ciencia. El otro reto tiene que ver con la necesidad de hacerse asesorar por especialistas en la materia que puedan orientar claramente sobre cómo ésta debe ser acotada y abordada; si es suficiente con un único diccionario de «ecología»o, en efecto, la disciplina necesita apellidos para ajustarse a su realidad actual -medio ambiente, contaminación, biodiversidad...-. Esto delimitaría la selección de entradas que, a su vez, podrían incluir marcas de subespecialidad que dejaran claras las disciplinas afines con las que se relaciona la ecología. Finalmente, resulta imprescindible que estos trabajos sean realizados, coordinados o asesorados por le- 
xicógrafos o terminólogos conocedores de las técnicas de elaboración de este tipo de obras de especialidad. Así se evitarían los errores y descuidos formales y conceptuales que hemos señalado y estas obras cumplirían con rigor los propósitos comunicativos y formativos para los que fueron realizadas. Este trabajo, al sacar a la luz las carencias, sólo ha pretendido modestamente contribuir en el progreso de esta actividad.

\section{BIBLIOGRAFÍA}

Ahumada Lara, I. (2001): «Problemas de la definición enciclopédica en las palabras especializadas». En: María Bargalló (et al.): Las lenguas de especialidad y su didáctica. Tarragona: Universitat Rovira i Virgili.

Azorín Fernández, D. Marimón Llorca, C., Martínez Linares, M. A., Santamaría Pérez, M. I. (2006): «Aspectos metodológicos para la elaboración de definiciones en diccionarios especializados». En: Actes de GLAT-BERTINORO 2006: Aspectos metodológicos para la elaboración de léxicos monolingües y multilingües. Boloña: Universidad de Boloña, 267-281.

Cabré, M. T. (1993): La terminología. Teoría, metodología, aplicaciones. Barcelona: Antártida/ Empúries.

Cabré, M. T., Feliu, J. (eds.) (2001): La terminología científico-técnica. Barcelona: IULA.

Cabré, M. T. (1999): La terminología: representación y comunicación. Barcelona: IULA.

Dirzo, R. (1990): «La biodiversidad como crisis ecológica actual ¿qué sabemos?». En: Ciencias, especial 4, 48-55.

Estopa R. (2001): «Elementos lingüísticos de las unidades terminológicas para su extracción automática». En: María Teresa Cabré, Judit Feliu (eds.) (2001): La terminología científico-técnica. Barcelona: IULA, 67-80.

Galán Rodríguez, C. (2003): «La ciencia en zapatillas: Análisis del discurso de divulgación científica». En: Anuario de Estudios Filológicos, 26, 137-156.

García Palacios, J. (2002): «El artículo lexicográfico en el diccionario de especialidad». En: Ignacio Ahumada Lara (coord.): Diccionarios y lenguas de especialidad. Jaén: Universidad de Jaén, $21-47$.

Lorente, M. (2001): «Teoría e innovación en terminografía: la definición terminográfica». En: María Teresa Cabré, Judit Feliu (eds.) (2001): La terminología científico-técnica. Barcelona: IULA, 81-112.

Margalef, R. (1993): Planeta azul, planeta verde. Barcelona: Prensa científica.

Martínez de Sousa, J. (2004): «La lexicografía especializada del español actual». En: Consuelo Gonzalo García, Valentín García Yebra: Manual de documentación y terminología para la traducción especializada. Madrid: Arco/libros, 173-190.

Martínez Linares, M ${ }^{a}$ A. (2007): La definición lexicográfica. Forma de la definición. Definición y contorno. En: www.liceus.com.

Porto Dapena, J. A. (2002): Manual de técnica lexicográfica. Madrid: Arco.

Sager, J. C. (1993): Curso práctico sobre el procesamiento de la terminología. Madrid: Fundación Germán Sánchez Ruipérez.

Santamaría Pérez, M. I. (2007): Los diccionarios especializados. En: www.liceus.com. 


\section{TABLA 1 - EVALUACIÓN DE LOS PRELIMINARES}

\begin{tabular}{|c|c|c|c|c|c|c|c|c|}
\hline & GVDEMA & GDMAC & DMA & DTCA & DTE & www.DE-S & www.DE & EURDAT \\
\hline Destinatarios & \begin{tabular}{|l|} 
- Sector \\
amplio de la \\
población \\
(ESO, BACH, \\
COU, $1^{\circ}$ s \\
cursos Univ.), \\
interesados sin \\
formación \\
científica
\end{tabular} & $\begin{array}{l}\text { - Todos los } \\
\text { relacionados con } \\
\text { actividades } \\
\text { medioambientales } \\
\text { (científicos, } \\
\text { docentes, } \\
\text { ingenieros, } \\
\text { traductores } \\
\text { abogados, } \\
\text { periodistas, etc) } \\
\text { - Bibliotecas, } \\
\text { empresas } \\
\text { multinacionales } \\
\text { multilingües. }\end{array}$ & $\begin{array}{l}\text { - Dirigido a todos } \\
\text { los que trabajan, } \\
\text { estudian o están } \\
\text { interesados en } \\
\text { temas medio } \\
\text { ambientales }\end{array}$ & $\begin{array}{l}\text { - Científicos, } \\
\text { técnicos, } \\
\text { miembros de } \\
\text { organizaciones. } \\
\text { Investigadores, } \\
\text { especialistas, } \\
\text { estudiantes, } \\
\text { profesores de la } \\
\text { Universidad y } \\
\text { legisladores } \\
\text { medioambientales. }\end{array}$ & & $\begin{array}{l}\text { - Ecólogos } \\
\text { latinoamericanos }\end{array}$ & & \\
\hline Propósito & $\begin{array}{l}\text { - La consulta } \\
\text { - Despertar } \\
\text { mayor interés } \\
\text { y conciencia- } \\
\text { ción por el } \\
\text { medio } \\
\text { ambiente }\end{array}$ & $\begin{array}{l}\text { - Facilitar los } \\
\text { contactos } \\
\text { internacionales en } \\
\text { el ámbito } \\
\text { medioambiental. } \\
\text { - Ofrecer los } \\
\text { términos en español } \\
\text { con traducción al } \\
\text { francés y al inglés. }\end{array}$ & $\begin{array}{l}\text { - Cubrir una } \\
\text { necesidad, dada la } \\
\text { multidisciplinarie- } \\
\text { dad de la materia }\end{array}$ & $\begin{array}{l}\text { - Debido a la gran } \\
\text { confusión term } \\
\text { inológica: ofrecer } \\
\text { una nomenclatura } \\
\text { apropiada con la } \\
\text { definición de los } \\
\text { términos más } \\
\text { usuales en } \\
\text { contaminación } \\
\text { ambiental } \\
\text { - Ayudar a la } \\
\text { puesta al día de } \\
\text { aspectos poco } \\
\text { considerados en } \\
\text { obras técnicas } \\
\text { - Retroajustar la } \\
\text { terminología. }\end{array}$ & & $\begin{array}{l}\text { - Robustecer la } \\
\text { disciplina en } \\
\text { Latinoamérica } \\
\text { cuyo material } \\
\text { está en inglés. } \\
\text { - Facilitar la } \\
\text { reestructuración } \\
\text { del estado del } \\
\text { conocimiento } \\
\text { ecológico en } \\
\text { nuestros países }\end{array}$ & & \\
\hline $\begin{array}{l}\text { Criterio de } \\
\text { selección de } \\
\text { entradas }\end{array}$ & \begin{tabular}{|l|} 
- 3000 \\
términos \\
- enfoque \\
global y \\
generalista. \\
- Se prescinde \\
de términos \\
muy \\
especializados \\
(quimicos) \\
- Centrado en \\
las palabras \\
propias de la \\
ecología \\
y el medio \\
ambiente \\
\end{tabular} & $\begin{array}{l}\text { - } 18.000 \text { términos } \\
\text { en español de un } \\
\text { total de } 60.000 \\
\text { - } 5000 \text { definiciones } \\
\text { elaboradas por el } \\
\text { propio equipo. } \\
\text { - Contiene el } \\
\text { vocabulario y la } \\
\text { terminología de } \\
\text { uso en las ciencias } \\
\text { del medio ambiente } \\
\text { - Selección de } \\
\text { voces más útiles y } \\
\text { practicas } \\
\text { - Incluye productos } \\
\text { químicos }\end{array}$ & $\begin{array}{l}\text { - Los términos } \\
\text { más importantes } \\
\text { - Se han incluido } \\
\text { aspectos de legis- } \\
\text { lación, corrientes } \\
\text { filosóficas, acuer- } \\
\text { dos, expresiones } \\
\text { cotidianas }\end{array}$ & $\begin{array}{l}\text { - La noción de } \\
\text { contaminación } \\
\text { ambiental, tomada } \\
\text { en sentido amplio. } \\
\text { - Incluye términos } \\
\text { definitorios en } \\
\text { inglés } \\
\text { - Incluye un } \\
\text { glosario español/ } \\
\text { inglés organizado } \\
\text { en entradas } \\
\text { principales y } \\
\text { secundarias }\end{array}$ & & $\begin{array}{l}\text { - Recopilación } \\
\text { restringida por } \\
\text { espacio y tiempo. } \\
\text { - Hay términos } \\
\text { reformulados y } \\
\text { adaptados al } \\
\text { español } \\
\text { latinoamericano } \\
\text { - Invita al lector } \\
\text { a incluir términos } \\
\text { - Proceden de } \\
\text { distintas ramas } \\
\text { de la disciplina }\end{array}$ & & \\
\hline Fuentes & & $\begin{array}{l}\text { - Cita los sectores } \\
\text { de los que se ha } \\
\text { extraído los } \\
\text { términos } \\
\text { - Lista de } \\
\text { organismos } \\
\text { consultados }\end{array}$ & $\begin{array}{l}\text { - Legislación, } \\
\text { corrientes } \\
\text { filosóficas, acuerdos } \\
\text { mundiales y } \\
\text { europeos, expresio- } \\
\text { nes cotidianas, } \\
\text { managment. }\end{array}$ & & & $\begin{array}{l}\text { - Términos } \\
\text { tomados } \\
\text { directamente de } \\
\text { autores }\end{array}$ & & \\
\hline Otras & \begin{tabular}{|l|} 
- Actualidad \\
y multidiscip- \\
linariedad del \\
tema del \\
medio \\
ambiente. \\
- Panorama \\
léxico en \\
continua \\
renovación
\end{tabular} & $\begin{array}{l}\text { - Enorme } \\
\text { actualidad del } \\
\text { medio ambiente y } \\
\text { la contaminación } \\
\text { - No enciclopédico } \\
\text { - Detalles del } \\
\text { equipo de redactores } \\
\text { - Incluye } \\
\text { bibliografía } \\
\text { - Prólogo } \\
\text { institucional }\end{array}$ & $\begin{array}{l}\text { Pluridisciplinarie- } \\
\text { dad del tema } \\
\text { - Contiene anexos } \\
\text { con acuerdos de } \\
\text { conferencias } \\
\text { internacionales } \\
\text { - Prólogo que } \\
\text { presenta la } \\
\text { colección }\end{array}$ & $\begin{array}{l}\text { - Tema de enorme } \\
\text { actualidad (medio } \\
\text { ambiente y } \\
\text { contaminación) } \\
\text { - Anexos: } \\
\text { acrónimos en } \\
\text { nglés y abreviaturas } \\
\text { en inglés. } \\
\text { - Incluye } \\
\text { bibliografía }\end{array}$ & & $\begin{array}{l}\text { - Capítulos } \\
\text { especiales con } \\
\text { términos no } \\
\text { traducidos del } \\
\text { inglés, raíces } \\
\text { griegas y latinas. } \\
\text { - Anexo con } \\
\text { organizaciones }\end{array}$ & & \\
\hline
\end{tabular}




\section{2 - TIPO DE INFORMACIÓN CONTENIDA EN EL ARTÍCULO}

\begin{tabular}{|l|c|c|c|c|c|c|c|c|}
\hline & GVDEMA & GDMAC & DMA & DTCA & DTE & www.DE-S & www.DE & EURDAT \\
\hline Etimología & $\mathrm{N}$ & $\mathrm{N}$ & $\mathrm{N}$ & $\mathrm{N}$ & $\mathrm{N}$ & $\mathrm{N}$ & $\mathrm{N}$ & $\mathrm{N}$ \\
\hline Nombre científico & $\mathrm{N}$ & $\mathrm{N}$ & $\mathrm{N}$ & $\mathrm{N}$ & $\mathrm{N}$ & $\mathrm{N}$ & $\mathrm{N}$ & $\mathrm{N}$ \\
\hline Categoría gramatical & $\mathrm{S}$ & $\mathrm{N}$ & $\mathrm{N}$ & $\mathrm{N}$ & $\mathrm{N}$ & $\mathrm{N}$ & $\mathrm{N}$ & $\mathrm{N}$ \\
\hline $\begin{array}{l}\text { Marcas temáticas } \\
\text { de subespecialidad }\end{array}$ & $\mathrm{S}$ & $\mathrm{N}$ & $\mathrm{N}$ & $\mathrm{N}$ & $\mathrm{N}$ & $\mathrm{N}$ & $\mathrm{N}$ & $\mathrm{N}$ \\
\hline Información gramatical & $\mathrm{N}$ & $\mathrm{N}$ & $\mathrm{N}$ & $\mathrm{N}$ & $\mathrm{N}$ & $\mathrm{N}$ & $\mathrm{N}$ & $\mathrm{N}$ \\
\hline $\begin{array}{l}\text { Equivalente en } \\
\text { otra lengua }\end{array}$ & $\mathrm{N}$ & $\mathrm{S}$ & $\mathrm{N}$ & $\mathrm{S}$ & $\mathrm{N}$ & $\mathrm{S}$ & $\mathrm{N}$ & $\mathrm{S}$ \\
\hline Sinónimos en la misma & $\mathrm{N}$ & $\mathrm{N}$ & $\mathrm{N}$ & $\mathrm{N}$ & $\mathrm{N}$ & $\mathrm{S}$ & $\mathrm{N}$ & $\mathrm{N}$ \\
\hline Información normativa & $\mathrm{N}$ & $\mathrm{N}$ & $\mathrm{N}$ & $\mathrm{N}$ & $\mathrm{N}$ & $\mathrm{N}$ & $\mathrm{N}$ & $\mathrm{N}$ \\
\hline Definición & $\mathrm{S}$ & $\mathrm{S}$ & $\mathrm{S}$ & $\mathrm{S}$ & $\mathrm{S}$ & $\mathrm{S}$ & $\mathrm{S}$ & $\mathrm{N}$ \\
\hline Ejemplos & $\mathrm{N}$ & $\mathrm{N}$ & $\mathrm{N}$ & $\mathrm{N}$ & $\mathrm{N}$ & $\mathrm{N}$ & $\mathrm{N}$ & $\mathrm{N}$ \\
\hline Iconoestructura & $\mathrm{S}$ & $\mathrm{N}$ & $\mathrm{N}$ & $\mathrm{N}$ & $\mathrm{N}$ & $\mathrm{N}$ & $\mathrm{N}$ & $\mathrm{N}$ \\
\hline
\end{tabular}

\section{3 - TIPOS DE DEFINICIÓN}

\begin{tabular}{|l|c|c|c|c|c|c|c|c|}
\hline & GVDEMA & GDMAC & DMA & DTCA & DTE & www.DE-S & www.DE & EURDAT \\
\hline $\begin{array}{l}\text { Analítica o perifrástica } \\
\text { (hiperonímica, } \\
\text { metonímica) }\end{array}$ & $\mathrm{S}$ & $\mathrm{S}$ & $\mathrm{S}$ & $\mathrm{S}$ & $\mathrm{S}$ & $\mathrm{S}$ & $\mathrm{S}$ & $\mathrm{S}$ \\
\hline $\begin{array}{l}\text { Sintética o descriptiva } \\
\text { (incluye información } \\
\text { enciclopédica) }\end{array}$ & $\mathrm{S}$ & $\mathrm{N}$ & $\mathrm{N}$ & $\mathrm{S}$ & $\mathrm{S}$ & $\mathrm{S}$ & $\mathrm{S}$ & $\mathrm{N}$ \\
\hline $\begin{array}{l}\text { Por extensión } \\
\text { o denotativa (ejemplos) }\end{array}$ & $\mathrm{S}$ & $\mathrm{N}$ & $\mathrm{S}$ & $\mathrm{S}$ & $\mathrm{N}$ & $\mathrm{S}$ & $\mathrm{S}$ & $\mathrm{N}$ \\
\hline Mediante sinónimos & $\mathrm{N}$ & $\mathrm{N}$ & $\mathrm{N}$ & $\mathrm{N}$ & $\mathrm{N}$ & $\mathrm{S}$ & $\mathrm{N}$ & $\mathrm{S}$ \\
\hline $\begin{array}{l}\text { Paráfrasis } \\
\text { (o explicación) }\end{array}$ & $\mathrm{N}$ & $\mathrm{N}$ & $\mathrm{S}$ & $\mathrm{N}$ & $\mathrm{N}$ & $\mathrm{S}$ & $\mathrm{N}$ & $\mathrm{N}$ \\
\hline Por implicación & $\mathrm{N}$ & $\mathrm{N}$ & $\mathrm{N}$ & $\mathrm{S}$ & $\mathrm{N}$ & $\mathrm{S}$ & $\mathrm{N}$ & $\mathrm{N}$ \\
\hline Por demostración & $\mathrm{N}$ & $\mathrm{N}$ & $\mathrm{N}$ & $\mathrm{N}$ & $\mathrm{N}$ & $\mathrm{N}$ & $\mathrm{N}$ & $\mathrm{N}$ \\
\hline Remisión & $\mathrm{S}$ & $\mathrm{N}$ & $\mathrm{N}$ & $\mathrm{S}$ & $\mathrm{S}$ & $\mathrm{N}$ & $\mathrm{S}$ & $\mathrm{N}$ \\
\hline
\end{tabular}

\section{EKOLOŠKI IN OKOLJSKI SLOVARJI V ŠPANŠČINI ALI BESEDIŠČE MED ZNANOSTJO IN ZAVESTJO}

Namen pričujočega prispevka je splošna presoja slovarjev o ekologiji in okolju in analiza iztočnic ter predlaganih definicij in podatkov v geselskih člankih. Ker gre za dela, ki so nastala $\mathrm{v}$ okviru te nove discipline, nam njihova analiza dovoljuje opazovati težave in omejitve, s katerimi se soočajo avtorji pri izdelavi takšnih slovarjev in leksikalnih specializiranih zbirk. To je pereče predvsem takrat, ko je vsebina - tak je tudi naš primer - hkrati predmet znanstvenega raziskovanja in razprave v družbi. Metodološko je delo narejeno primerjalno na podlagi osmih specializiranih leksikografskih del, ki obravnavajo ekologijo ali eno od sorodnih disciplin. 